\title{
Erratum: "Calculation of the Electronic Structure
} of Metal Island Films" [Journal of Surface Investigation. X-Ray, Synchrotron and Neutron Techniques 4, 672 (2011)]

\author{
E. R. Amanbaev, E. Yu. Zykova, A. A. Klavsyuk, T. N. Polivnikova, \\ A. A. Khaidarov, and A. L. Klavsyuk \\ Physics Faculty, Moscow State University, Moscow, 119899 Russia \\ e_mail:Ivan.Gainullin@gmail.com \\ (Submitted August 24, 2011; accepted for publication August 24, 2011)
}

DOI: $10.1134 / \mathrm{S} 1027451011130015$

The list of the authors should read as follows:

E. R. Amanbaev, E. Yu. Zykova, A. L. Klavsyuk, T. N. Polivnikova, A. A. Khaidarov, I. K. Gainullin 